\title{
Childhood onset of Scheie syndrome, the attenuated form of mucopolysaccharidosis I
}

\author{
Janet A. Thomas $\cdot$ Michael Beck • Joe T. R. Clarke • \\ Gerald F. Cox
}

Received: 15 January 2010 /Revised: 31 March 2010 /Accepted: 12 April 2010 /Published online: 2 June 2010

(C) The Author(s) 2010. This article is published with open access at Springerlink.com

\begin{abstract}
Scheie syndrome is the most attenuated and rarest form of mucopolysaccharidosis type I (MPS I), an inherited lysosomal storage disorder. Only small patient series have previously been reported. Using natural history data from the uniquely large population of 78 Scheie patients enrolled in the MPS I Registry, we characterized the onset and prevalence of clinical manifestations and explored reasons for delayed diagnosis of the disease. Median patient age was 17.5 years; $46 \%$ of the patients were male, and $88 \%$ were Caucasian. Of 25 MPS I-related
\end{abstract}

Communicated by: Ed Wraith

References to electronic databases: Mucopolysaccharidosis type 1: OMIM\#252800. $\alpha$-L-iduronidase: EC 3.2.1.76.

Competing interests: The MPS I Registry is sponsored by Genzyme Corporation. J.A. Thomas is a member of the North American Board of Advisors for the MPS I Registry and recipient of an educational grant from Genzyme. M. Beck is a member of the European Board of Advisors for the MPS I Registry and recipient of honoraria, consultancy fees, speaker's bureau fees, and study funding from Genzyme. J.T.R. Clarke is a member of the North American Board of Advisors for the MPS I Registry. G.F. Cox is an employee and stockholder of Genzyme.

\section{J. A. Thomas}

University of Colorado,

Denver, CO, USA

M. Beck

Children's Hospital, University of Mainz,

Mainz, Germany

\section{J. T. R. Clarke}

Hospital for Sick Children,

Toronto, ON, Canada

G. F. Cox

Genzyme Corporation,

Cambridge, MA, USA clinical features, cardiac valve abnormalities, joint contractures, and corneal clouding were each reported by $>80 \%$ and all three by $53 \%$ of patients. Carpal tunnel syndrome, hernia, coarse facial features, and hepatomegaly were each reported by $>50 \%$ of patients. Age at onset of the clinical features varied widely between individuals, but the median age at onset was 3 years for hernia and between 5 and 12 years for most features, including coarse facial features, hepatomegaly, joint contractures, bone deformities, cardiac valve abnormalities, cognitive impairment, and corneal

G. F. Cox

Children's Hospital Boston,

Boston, MA, USA

G. F. Cox

Harvard Medical School,

Boston, MA, USA

\section{J. A. Thomas $(\bowtie)$}

Department of Pediatrics, University of Colorado,

13123 East 16th Avenue, B-153, Mail Stop 8400,

Aurora, CO 80045, USA

e-mail: thomas.janet@tchden.org 
clouding. Carpal tunnel syndrome, cardiomyopathy, and myelopathy arose more commonly during adolescence or adulthood. Delays up to 47 years intervened between symptom onset and disease diagnosis, and the longest delays were associated with later age at symptom onset and symptom onset before 1980. In summary, Scheie syndrome usually emerges during childhood, and recognition of attenuated MPS I requires awareness of the multisystemic disease manifestations and their diverse presentation. Given the availability of etiologic treatment, prompt diagnosis is important.

\section{Abbreviations \\ MPS I Mucopolysaccharidosis type 1 \\ ERT Enzyme replacement therapy \\ GAG Glycosaminoglycan(s) \\ HSCT Hematopoietic stem cell transplantation \\ IDUA $\alpha$-L-iduronidase}

\section{Introduction}

Timely recognition and accurate diagnosis of Scheie syndrome, the attenuated form of mucopolysaccharidosis type I (MPS I; OMIM\#252800), have acquired greater urgency with the advent of enzyme replacement therapy (ERT) as an etiologic treatment option for this rare lysosomal storage disorder (Clarke et al. 2009; Giugliani et al. 2009; Kakkis et al. 2001; Wraith et al. 2004). MPS I results from the autosomal recessive inheritance of a deficiency of $\alpha$-L-iduronidase (IDUA; EC 3.2.1.76), an enzyme in the degradative pathway for two glycosaminoglycans (GAG), heparan sulfate and dermatan sulfate, which contribute to the structural and functional integrity of most connective tissues. IDUA deficiency leads to widespread GAG accumulation accompanied by deterioration of function in multiple organ systems (Neufeld and Muenzer 2001). The most prominent and debilitating manifestations usually arise in the musculoskeletal, cardiorespiratory, and central nervous systems, but individual patients vary considerably in the timing of symptom onset, the pattern of organ involvement, and the rate of disease progression. Three syndromes, broadly based on the severity of clinical complications and age at presentation, are used to categorize the heterogeneous MPS I phenotypes. Patients expressing the most severe phenotype, Hurler syndrome, rapidly develop conspicuous signs, including characteristic facial dysmorphia and progressive cognitive impairment, that lead to disease diagnosis within the first 2 years of life. In contrast, patients with the most attenuated phenotype, Scheie syndrome, manifest variable and generally milder symptoms that may emerge over many years, often delaying diagnosis. The third syndrome, Hurler-Scheie, designates an intermediate phenotype and underscores that MPS I spans a continuous disease spectrum without clear lines of demarcation between the three syndromes.

Due to its rarity, Scheie syndrome has been described in small case series only, with patients selected on the basis of a single clinical parameter or combined with other MPS I phenotypes (Cimaz et al. 2006; Scheie et al. 1962; Stevenson et al. 1976; Vieira et al. 2008; Vijay and Wraith 2005). A uniquely large Scheie population is now available for analysis from the MPS I Registry (Pastores et al. 2007), a global observational program for monitoring patients with MPS I (www.mpsiregistry.com). Here we use natural history data from 78 registry patients diagnosed with Scheie syndrome to characterize the onset and prevalence of disease manifestations and to explore reasons why diagnosis is often delayed.

\section{Methods}

The MPS I Registry was established in 2003 for the longterm observation of MPS I patients in compliance with regulatory commitments upon marketing approval of ERT with laronidase, a recombinant form of human IDUA (Aldurazyme ${ }^{\circledR}$; BioMarin Pharmaceutical Inc., Novato, CA, USA, and Genzyme Corporation, Cambridge, MA, USA). Any patient with a confirmed MPS I diagnosis can be enrolled in the registry by a treating physician. Patient authorization that complies with local and international guidelines is obtained prior to enrollment. Registry data are derived from responses to a detailed questionnaire for monitoring MPS I-related clinical signs based on medical history and ongoing assessments (Pastores et al. 2007). Individual patient data remain confidential.

Analyses of MPS I Registry data collected from October 2003 to October 2008 were performed by Biomedical Data Sciences and Informatics at Genzyme. A total of 759 patients were enrolled, and syndrome diagnoses were provided by the treating physician as follows: $78(10.3 \%)$ with Scheie syndrome, 292 (55.7\%) with Hurler syndrome, and 180 (23.7\%) with Hurler-Scheie syndrome; for the remaining $10.3 \%$, a syndrome designation was undetermined or missing. For the analyses reported here, the study population consisted of all patients with an entered diagnosis of Scheie syndrome. The 78 patients with Scheie syndrome were enrolled by 38 physicians from 17 countries in North America, Europe, Latin America, and Asia Pacific. Each physician enrolled between one and eight patients, with two physicians enrolling seven and eight patients, respectively. Only natural history data [before ERT initiation or hematopoietic stem cell transplanta- 
tion (HSCT)] were evaluated for the Scheie cohort. Individuals were excluded from an analysis if relevant entries were missing or unknown. Patient numbers are stated for each analysis, and descriptive statistics are presented. Pearson's correlation coefficients $(r)$, computed to assess associations between parameters, were considered statistically significant if $P$ values derived from $t$ tests were $<0.05$.

\section{Results}

\section{Patient population}

Demographic data are presented in Table 1 for the 78 patients with Scheie syndrome enrolled in the MPS I Registry. Diagnostic IDUA enzyme activity levels or IDUA gene mutation(s) were reported for each patient, but initial MPS I diagnoses were based on some combination of clinical presentation $(n=55)$, enzyme activity $(n=48)$, and/ or gene mutations $(n=5)$. A total of 24 patients reported siblings with MPS I, but families were not linked in the registry at the time of analysis. Two patients were enrolled posthumously: a 23-year-old man who died of heart failure and cor pulmonale, and a 17-year-old woman who died of multiorgan failure and septicemia 11 days after mitral and aortic valve replacement surgery.

\section{Clinical features}

Figure 1 demonstrates that 19 of the 25 clinical features monitored in the registry arose during childhood in the majority of patients, although wide variability was observed

Table 1 Demographic data for patients with Scheie syndrome

\begin{tabular}{ll}
\hline Parameter & Value $[n(\%)]$ \\
\hline Patients with Scheie syndrome & 78 \\
Alive & $76(97.4)$ \\
Deceased & $2(2.6)$ \\
Gender & \\
Male & $36(46.2)$ \\
Female & $42(53.8)$ \\
Ethnicity & \\
Caucasian & $69(88.5)$ \\
Black & $2(2.6)$ \\
Hispanic & $1(1.3)$ \\
Asian & $1(1.3)$ \\
Other & $5(6.4)$ \\
Age in years & \\
Mean (standard deviation) & $22.9(13.6)$ \\
Median & 17.5 \\
Minimum, maximum & $1.8,62.9$ \\
\hline
\end{tabular}

between individual patients for each feature. Hernia was the only feature with a median age at onset before 5 years, whereas a median age at onset between 5 and 12 years was observed for 18 clinical features, mostly involving infiltration and enlargement of soft tissues or joint and bone complications. Scoliosis, carpal tunnel syndrome, and congestive heart failure were more likely to arise in adolescence; and glaucoma, cardiomyopathy, and myelopathy in early adulthood. Based on the onset dates entered for the 25 clinical features, none emerged as a first sign in a majority of patients. Hernia and joint contractures each appeared as a first sign in approximately $30 \%$ of the 72 patients with data, but hernia was more often reported as an isolated finding at birth or within the first year of life, whereas joint contractures usually appeared after age 2 and more often in conjunction with other disease manifestations.

The median number of clinical features reported per patient was seven (range 0-15). The five most prevalent were cardiac valve abnormalities, joint contractures, corneal clouding, carpal tunnel syndrome, and hernia (Fig. 2). All five features were reported by $31.0 \%$ of patients; and four, three, two, or one feature was reported, respectively, by $18.3 \%, 25.4 \%$, $14.1 \%$, or $5.6 \%$. Only $5.6 \%$ reported none of the five.

Cardiac valve abnormalities were reported by $87.9 \%$ (58/ 66) of patients at a median age of 11.7 years. Echocardiography data available for 50 patients showed abnormal function of one valve in $40 \%$ and of two or more valves in $60 \%$. The mitral and aortic valves were most commonly affected (Table 2), with valve replacement surgery documented for $14 \%$ of the patients.

Joint contractures were reported in $87.7 \%(57 / 65)$ of patients at a median age of 7.2 years. Joint contractures, frequently observed in conjunction with other musculoskeletal abnormalities, were documented for $>90 \%$ of patients with pes cavus, genu valgum, and toe walking and by $84-87 \%$ of patients with dysostosis multiplex, kyphosis gibbus, scoliosis, and hip dysplasia. Among 71 patients reporting clinical features, tendon release surgery was reported for $4 \%$; hip, knee, or foot surgery for $8 \%$; and spine surgery for $4 \%$.

Corneal clouding was reported by $81.8 \%$ (54/66) of patients at a median age of 9.1 years. Results of slit-lamp examinations available for 32 patients revealed that corneal clouding occurred bilaterally in all patients and was of equal severity in both eyes except in one patient. The degree of clouding, considered mild in $78.1 \%(25 / 32)$ of patients, did not correlate with age at assessment.

Carpal tunnel syndrome was reported by $66.7 \%$ (44/66) of patients at a median age of 13.1 years. Surgical interventions were documented for $52.2 \%$ (23/44) of those affected. Accompanying symptoms reported by 35 patients included pain $(71.4 \%)$, tingling $(54.2 \%)$, numbness $(51.4 \%)$, weakness $(34.3 \%)$, and burning sensation $(20.0 \%)$.

Hernia was reported for $65.1 \%(41 / 63)$ of patients at a median age of 3.3 years. Among 39 patients with data 
Fig. 1 Age at onset of 25 clinical features of mucopolysaccharidosis type I (MPS I). The symbol represents the median; the bar shows the range; $n=$ the number of patients with an onset date reported

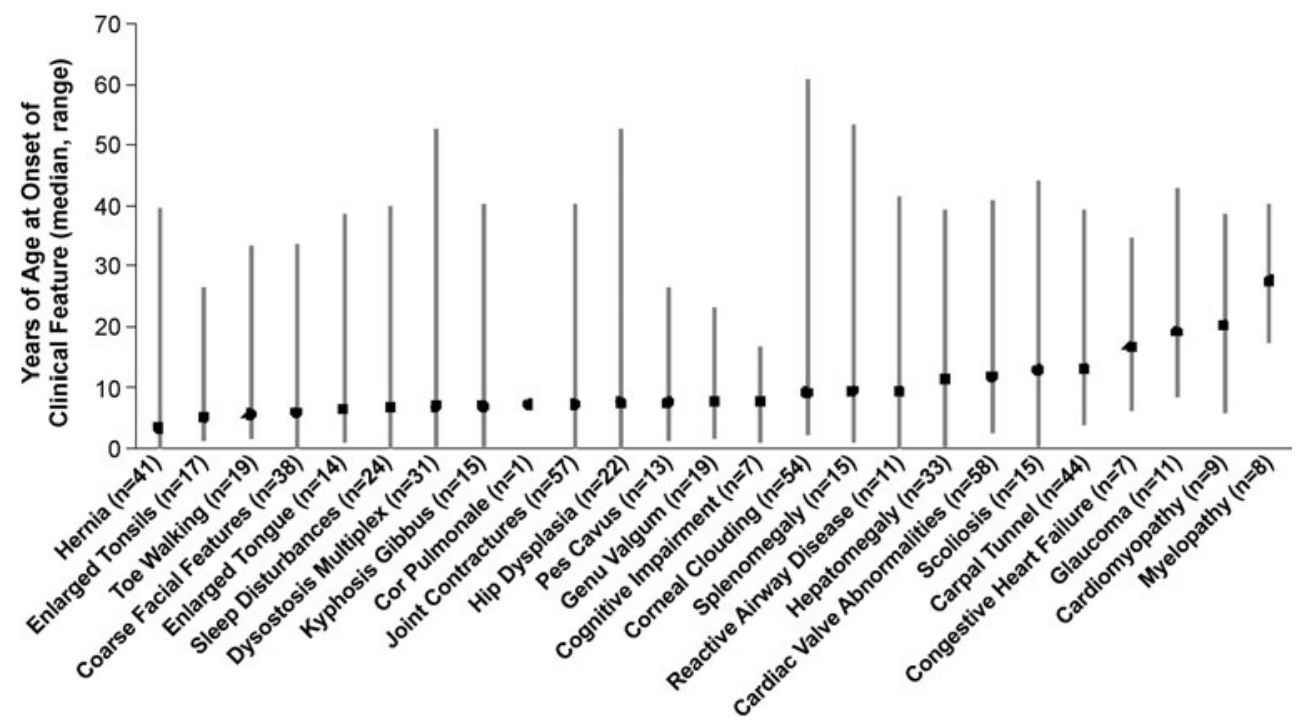

entered, umbilical hernias occurred more commonly $(87.2 \%)$ than left $(30.8 \%)$ and right $(25.6 \%)$ inguinal hernias. The majority $(66.7 \%)$ reported hernia in a single location; $10.2 \%$ and $17.9 \%$ had hernias in two and three locations, respectively. Herniorrhaphy was documented for $68.3 \%(28 / 41)$.

Diagnostic delays

For 71 patients with dates reported, age at first symptom onset ranged from birth to 33.8 (median 5.4) years, and age of MPS I diagnosis ranged from before birth to 54.1 (median 9.8) years. Two patients, both with MPS I siblings, were diagnosed presymptomatically: one before birth and one at age 5 years. The remaining 69 patients were diagnosed after symptom onset, with delays of 0 to
47.2 (median 2.0) years from first symptom onset to disease diagnosis. To explore differences in diagnostic delays, the 69 patients were categorized by age at diagnosis into four subgroups. Figure $3 \mathrm{a}$ demonstrates that the median age at first symptom onset was younger than 10 years for all subgroups, but the range broadened as age at diagnosis increased. The delay from first symptom onset to disease diagnosis was longer and more variable as age at diagnosis increased (Fig. 3a). The median number of clinical features present at diagnosis did not differ among subgroups (three for each subgroup diagnosed before age 20; five for the subgroup diagnosed after age 20), but differences between subgroups were noted in the most prevalent clinical features present at diagnosis (Fig. 3b). Coarse facial features was reported by $63 \%$ of the subgroup diagnosed before age 5 years compared with $21-31 \%$ of
Fig. 2 Percentage of patients with Scheie syndrome reporting clinical features of mucopolysaccharidosis type 1 (MPS I). Percentages, calculated from the fractions shown in parentheses, represent the number of patients who entered "yes" divided by the sum of those who entered "yes" or "no" for the presence of the clinical feature

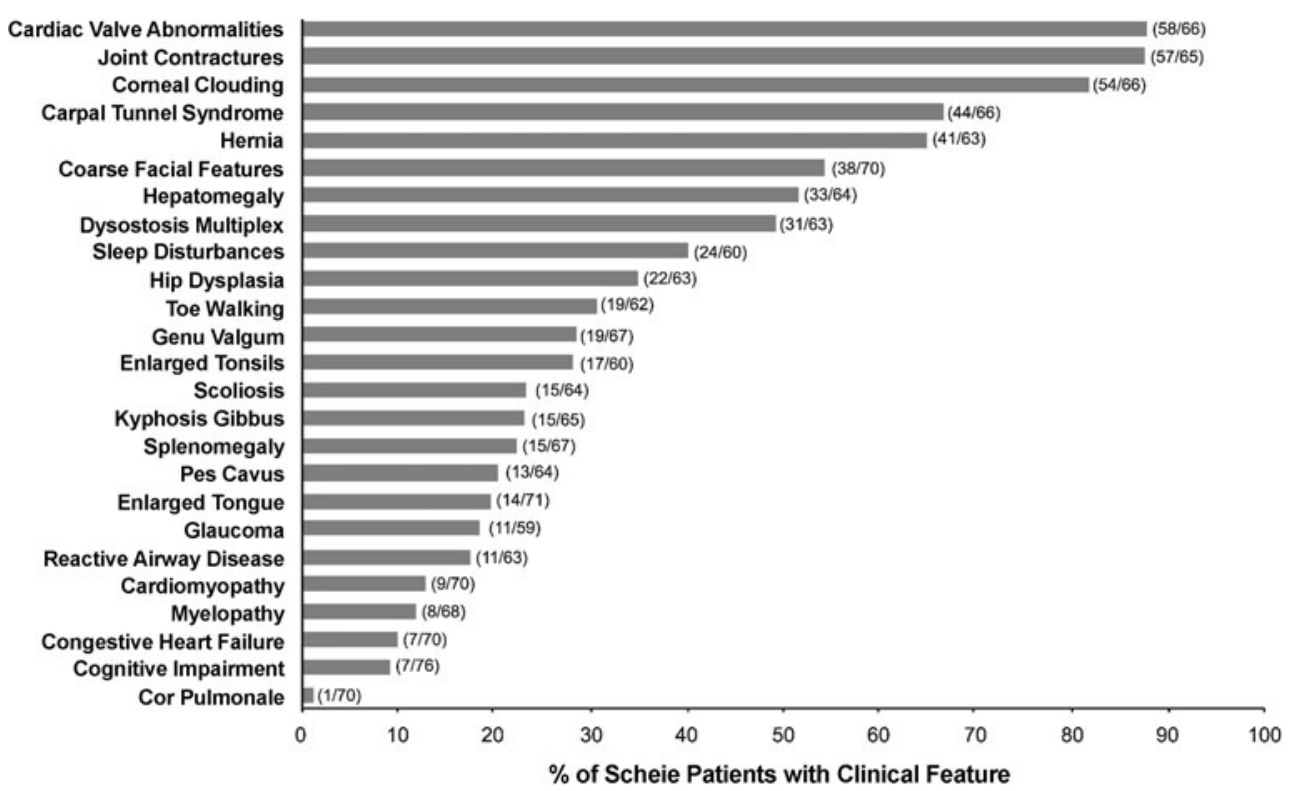


Table 2 Cardiac valve abnormalities detected by echocardiography in patients with Scheie syndrome

Total number of patients evaluated: 50

\begin{tabular}{llll}
\hline $\begin{array}{l}\text { Stenosis } \\
{[\%(n)]}\end{array}$ & $\begin{array}{l}\text { Regurgitation } \\
{[\%(n)]}\end{array}$ & $\begin{array}{l}\text { Prolapse } \\
{[\%(n)]}\end{array}$ & $\begin{array}{l}\text { Any } \\
{[\%(n)]}\end{array}$ \\
\hline $32(16)$ & $76(38)$ & $16(8)$ & $78(39)$ \\
$36(18)$ & $56(28)$ & $\mathrm{n} / \mathrm{a}$ & $66(33)$ \\
0 & $24(12)$ & $\mathrm{n} / \mathrm{a}$ & $24(12)$ \\
$4(2)$ & $8(4)$ & $\mathrm{n} / \mathrm{a}$ & $12(6)$
\end{tabular}

$n / a$ not applicable

subgroups diagnosed after age 5 years. Joint contracture was most prevalent in the subgroup diagnosed between 5 and 10 years of age, and dysostosis multiplex and cardiac valve abnormalities were most common in the subgroup diagnosed after age 20 years. Figure $3 \mathrm{c}$ indicates that clinical manifestations tended to arise earlier in patients diagnosed at younger ages and later in patients diagnosed at older ages. There were strong, statistically significant correlations between age at diagnosis and age at onset for all clinical features presented in Fig. 3c $(r=0.73-$ $0.98 ; P \leq 0.001)$, except hernia $(r=0.47 ; P=0.17)$.
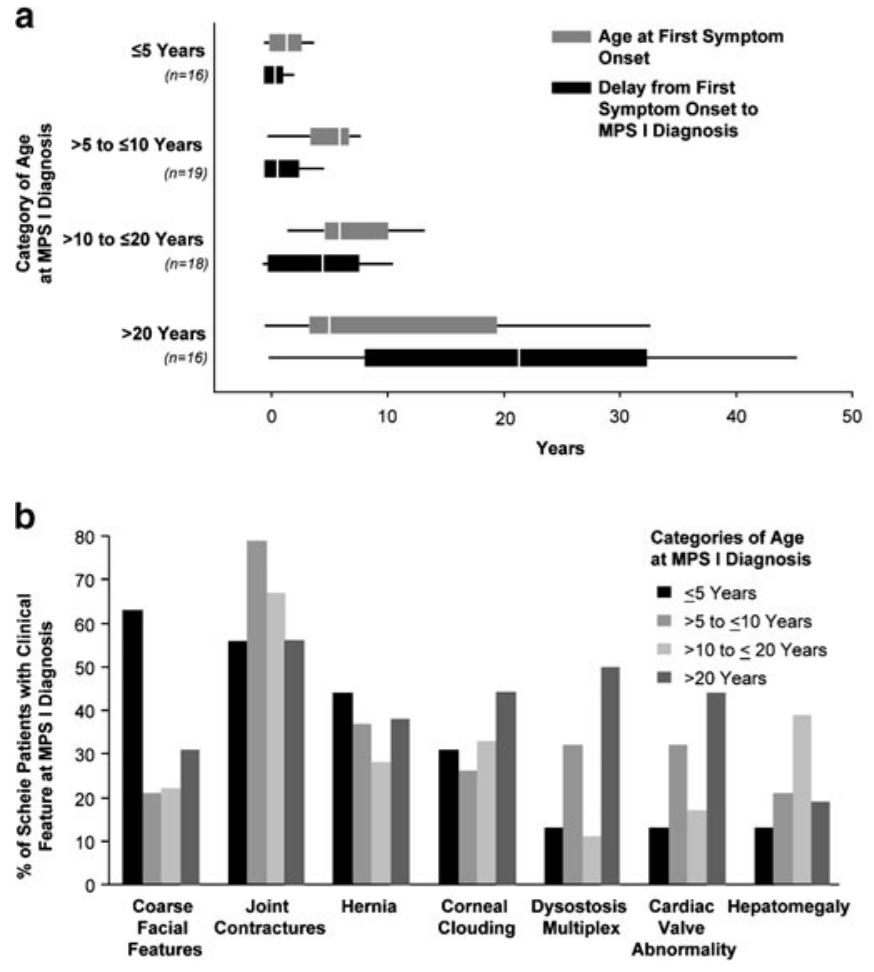

Fig. 3 Analyses to explore diagnostic delays. a Age at first symptom onset (upper box plot) and the interval from first symptom onset to diagnosis (lower box plot) for patients categorized by age at diagnosis (see $y$-axis). Data shown are the median (gap within box), 25th and 75th percentiles (left and right sides of box, respectively), and range (line extending from box). b The most prevalent clinical features
Figure $3 d$ displays the length of diagnostic delays as a function of the calendar year in which symptoms were first noted. Patients with the longest diagnostic delays had first symptom onset before 1980 .

\section{Discussion}

Natural history data were reviewed for 78 patients with Scheie syndrome, representing the largest cohort with this rare genetic disorder examined to date. Strikingly, the pattern of disease progression indicates that most clinical features associated with MPS I first appeared before the age of 12 years in the majority of patients. Among individuals, the first clinical feature(s) to emerge varied, but the most common first sign to appear before age 2 years was hernia and after age 2 years joint contractures. Some early signs, such as hernias and enlarged tonsils, are relatively common in the general pediatric population and in isolation would not raise suspicion for MPS I. More indicative of MPS I are the flexion contractures, especially of the fingers and hands, that children with Scheie syndrome often develop. In MPS I, joint pain, stiffness, and immobility are not accompanied by inflammation, as in some other rheumatological con-
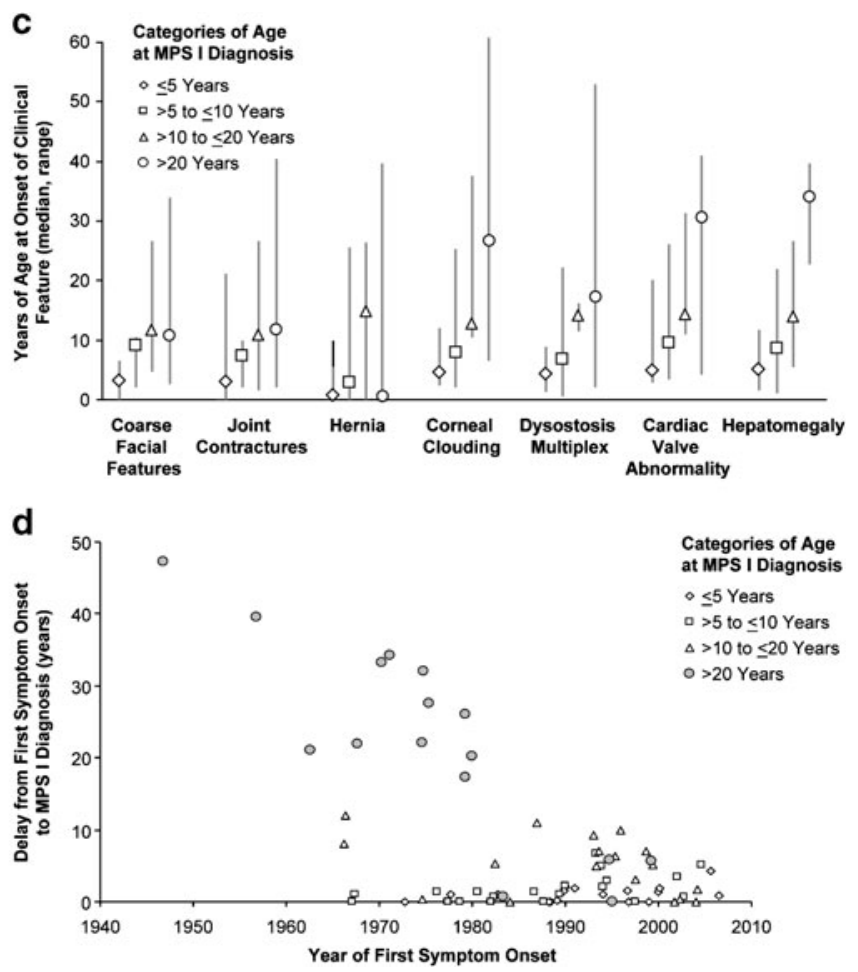

reported before diagnosis in patients categorized by age at diagnosis. $\mathbf{c}$ Age at onset of most prevalent clinical features in patients categorized by age at diagnosis. Symbols represent medians; lines represent ranges. d Trends in diagnosis over time in patients categorized by age at diagnosis. Each symbol represents a patient 
ditions that arise in childhood (Cimaz et al. 2006). The wide variability observed in the age at onset for each clinical feature underscores the heterogeneity of Scheie syndrome, with disease pathology emerging in individual patients at different times in different organs. While there is no typical clinical profile for the Scheie phenotype, cardiac valve abnormalities, joint contractures, and corneal clouding were each reported by $>80 \%$ of patients. Only $7 \%$ had none of these signs, whereas $53 \%$ reported all three. In 1962, Dr. Harold Scheie, an ophthalmologist, provided the first biochemical evidence linking the corneal haze he observed in ten patients to an attenuated forme fruste of Hurler syndrome (Scheie et al. 1962). Flexion contractures and heart murmurs were frequently noted among his patients. Recent reports emphasize that children and adults with Scheie syndrome most often present with rheumatological symptoms, usually accompanied by ocular and cardiac valve pathology (Cimaz et al. 2006; Vieira et al. 2008; Vijay and Wraith 2005). In addition to the triad of joint, cardiac valve, and corneal abnormalities, the majority of the registry patient cohort reported carpal tunnel syndrome, hernia, coarse facial features, and hepatomegaly. Chronic ear, nose, and throat and respiratory problems, including sleep apnea and recurrent otitis media, also have been noted in patients (Vijay and Wraith 2005; Wraith 2005). Furthermore, MPS I patients commonly require multiple, often unrelated, surgical interventions, and $40 \%$ of children with Scheie syndrome reported two or more surgeries by 10 years of age (Arn et al. 2009).

Following the onset of signs and symptoms, MPS I diagnosis was established for the registry patients with Scheie syndrome between infancy and the sixth decade. Analyses performed to explore this broad range suggested that patients diagnosed early express phenotypes that are more pronounced and that evolve more rapidly than patients diagnosed later. Prior to diagnosis, the facial dysmorphia that is characteristic of MPS I appeared in almost two thirds - and joint contractures in more than half - of the patients diagnosed before age 5 years. Among patients diagnosed after age 5, joint contractures occurred in the majority, but less than one third displayed coarse facial features before diagnosis. Patients diagnosed after age 20 years had, on average, slightly more clinical signs of MPS I at the time of diagnosis than patients diagnosed earlier, but no feature predominated as in the subgroups diagnosed earlier. For most clinical features, strong correlations were found between the age at which the feature was reported and the age at which the patient was diagnosed. Taken together, these observations suggest that MPS I manifestations emerged more slowly and variably in patients diagnosed after the age of 20 years. Yet, it is also noteworthy that the number of patients diagnosed has increased annually since 1962, when Scheie syndrome was first described, most likely mirroring a growing awareness of the attenuated phenotype. For the registry patients with Scheie syndrome born before 1970 and diagnosed in adulthood despite symptom onset in childhood, the lack of disease awareness is most likely responsible for the delayed diagnosis.

The limitations in interpreting the data presented here begin with the diagnosis of Scheie syndrome. MPS I spans a continuum of disease, and the three syndromes are not rigorously defined and cannot currently be distinguished by biochemical criteria or genotype in most cases. Hence, syndrome designation depends on the clinical judgment of the reporting physician. Subgroup analyses presented in Fig. 3 emphasize the likelihood that some registry patients express phenotypes that border the intermediate, HurlerScheie part of the disease spectrum. Indeed, two patients who were diagnosed before 18 months of age had coarse facial features and cognitive impairment present at diagnosis. Although early onset of these manifestations is more typical of Hurler or Hurler-Scheie syndromes, data are included from one patient up to 10.3 years of age when ERT was initiated and for the other up to 1.8 years of age when HSCT was performed. Another limitation of the reported data stems from the fact that MPS I is rare, and the attenuated phenotype is the rarest form of the disease (Meikle et al. 1999; Neufeld and Muenzer 2001). Even though the registry cohort is the largest examined thus far, the sample size is relatively small. Finally, limitations associated with any voluntary, observational disease registry must be considered, including selection bias, reporting bias, and unverified data entries (Pastores et al. 2007).

Clinical management of patients with Scheie syndrome requires an individualized approach best coordinated by a multidisciplinary team (Muenzer et al. 2009). ERT is approved in more than 55 countries worldwide for treatment of the non-neurological manifestations of MPS I. A phase 3 extension trial demonstrated stabilization or improvement of several clinical parameters in Scheie and Hurler-Scheie patients after approximately 4 years of ERT (Clarke et al. 2009). Improvement of cardiomyopathy with ERT has been reported, but cardiac valve disease appears to be less responsive, possibly because the treatment period was too short or because treatment was initiated late in the disease process (Braunlin et al. 2006). A recent case report of two siblings with Hurler-Scheie syndrome who began ERT at 5 months and 5 years of age, respectively, suggests that many MPS I disease manifestations, including cardiac valve disease, can be prevented if ERT is begun at an early age, but only partially reversed or stabilized if begun at a later age (Gabrielli et al. 2009). Thus, ERT is expected to provide the greatest clinical benefit when initiated before permanent tissue damage has ensued and attention has been drawn to patients with Scheie syndrome whose disease may 
not be recognized. Timely disease recognition also provides potential benefits with regard to genetic counseling for families and more timely interventions for patients, which, in turn, may produce better outcomes, slow disease progression, and/or improve quality of life. As reported here, clinical signs usually emerge during childhood in the attenuated phenotype, and prompt diagnosis requires awareness of the multisystemic manifestations and diverse presentation of MPS I.

Acknowledgements The contributions of patients and physicians who participate in the MPS I Registry are gratefully acknowledged. The MPS I Registry is sponsored by Genzyme Corporation, which provided support for data analyses by Gregory Fagan, Jessica Kong, and Lakshmi Rangachari, and manuscript preparation by Andrea Norfleet.

Open Access This article is distributed under the terms of the Creative Commons Attribution Noncommercial License which permits any noncommercial use, distribution, and reproduction in any medium, provided the original author(s) and source are credited.

\section{References}

Arn P, Wraith JE, Underhill L (2009) Characterization of surgical procedures in patients with mucopolysaccharidosis type I: findings from the MPS I Registry. J Pediatr 154(6):859-864, e853

Braunlin EA, Berry JM, Whitley CB (2006) Cardiac findings after enzyme replacement therapy for mucopolysaccharidosis type I. Am J Cardiol 98(3):416-418

Cimaz R, Vijay S, Haase C et al (2006) Attenuated type I mucopolysaccharidosis in the differential diagnosis of juvenile idiopathic arthritis: a series of 13 patients with Scheie syndrome. Clin Exp Rheumatol 24(2):196-202

Clarke LA, Wraith JE, Beck M et al (2009) Long-term efficacy and safety of laronidase in the treatment of mucopolysaccharidosis I. Pediatrics 123(1):229-240
Gabrielli O, Clarke LA, Bruni S, Coppa GV (2009) Enzymereplacement therapy in a 5-month-old boy with attenuated presymptomatic MPS I: 5-year follow-up. Pediatrics 125(1): e183-e187

Giugliani R, Rojas VM, Martins AM et al (2009) A dose-optimization trial of laronidase (Aldurazyme) in patients with mucopolysaccharidosis I. Mol Genet Metab 96(1):13-19

Kakkis ED, Muenzer J, Tiller GE et al (2001) Enzyme-replacement therapy in mucopolysaccharidosis I. N Engl J Med 344(3):182188

Meikle PJ, Hopwood JJ, Clague AE, Carey WF (1999) Prevalence of lysosomal storage disorders. JAMA 281(3):249-254

Muenzer J, Wraith JE, Clarke LA (2009) Mucopolysaccharidosis I: management and treatment guidelines. Pediatrics 123(1):19-29

Neufeld EF, Muenzer J (2001) The mucopolysaccharidoses. In: Scriver C, Beaudet A, Sly W et al (eds) The metabolic and molecular bases of inherited disease, 8th edn. McGraw-Hill, New York, pp 3421-3452

Pastores GM, Arn P, Beck M et al (2007) The MPS I registry: design, methodology, and early findings of a global disease registry for monitoring patients with Mucopolysaccharidosis Type I. Mol Genet Metab 91(1):37-47

Scheie HG, Hambrick GW Jr, Barness LA (1962) A newly recognized forme fruste of Hurler's disease (gargoylism). Am J Ophthalmol 53:753-769

Stevenson RE, Howell RR, McKusick VA et al (1976) The iduronidase-deficient mucopolysaccharidoses: clinical and roentgenorgraphic features. Pediatrics 57(1):111-122

Vieira T, Schwartz I, Munoz V et al (2008) Mucopolysaccharidoses in Brazil: what happens from birth to biochemical diagnosis? Am J Med Genet A 146A(13):1741-1747

Vijay S, Wraith JE (2005) Clinical presentation and follow-up of patients with the attenuated phenotype of mucopolysaccharidosis type I. Acta Paediatr 94(7):872-877

Wraith JE (2005) The first 5 years of clinical experience with laronidase enzyme replacement therapy for mucopolysaccharidosis I. Expert Opin Pharmacother 6(3):489-506

Wraith JE, Clarke LA, Beck M et al (2004) Enzyme replacement therapy for mucopolysaccharidosis I: a randomized, doubleblinded, placebo-controlled, multinational study of recombinant human alpha-L-iduronidase (laronidase). J Pediatr 144(5):581588 